\title{
Phyto-mediated Synthesis of Copper
} Nanoparticles by Cassia auriculata and its Characterization with reference to E-waste Management

\author{
Eswara Priya B, Sobiya S, Akshayalakshmi S M, Umamaheshwari S, Priyadharshini M, \\ Rathi Devi P
}

Department of Microbiology \& Clinical Lab Technology, Dr.Umayal Ramanathan College for Women, Karaikudi- 630003, Sivaganga Dt, TamilNadu, India.

\begin{abstract}
An eco-friendly loom has been taking up in the present study to synthesize copper nanoparticles using Cassia auriculata. The leaf extract of Cassia auriculata acts as reducing as well as capping agent. Synthesis of copper nanoparticles was initially confirmed by the visual observation i.e color change (dark green color). The synthesized copper nanoparticles were primarily characterized by UV-vis spectroscopy and Fourier Transform Infrared (FTIR) spectroscopy. Further, the formation of amorphous and crystalline phase was analyzed by X-Ray Diffraction pattern. The size and morphology of the synthesized Copper nanoparticles was characterized by Scanning Electron Microscopy (SEM) and the elemental composition was analyzed by EDAX. The present study is a preliminary investigation to know about the capability of Cassia auriculata to synthesize copper nanoparticles from its salts. The results of the present study confirmed that the leaf extract of Cassia auriculata be capable of recovering copper from printed circuit boards in the form of nanoparticles in near future.
\end{abstract}

Keywords- Copper Nanoparticles; Cassia auriculata; Nanotechnology; Characterization and E-waste.

\section{INTRODUCTION}

Electronic waste is the fastest escalating wastes in all over the world. E-waste consists of heavy metals such as silicon, arsenic, iron, copper, aluminium, lead, zinc, chromium, cadmium, mercury and barium. In addition most of these components can be recycled and reused [1,2]. Several techniques especially landfill and incineration has been applied to an E-waste management and both are causing considerable contamination risks [3,4]. An alternate method needed for the better E-waste management. Nanotechnology plays a key role in the recycling of E-waste through environment friendly manner [5]. Biological approaches using plants extracts and microorganisms for the extracting of metals have been recommended as expensive alternatives to conventional methods $[6,7,8]$.

Keeping the above all in view the present study is initially aimed to screen the ability of Cassia auriculata for the synthesis of copper nanoparticles from its salts. After confirming the capability of Cassia auriculata to synthesize copper nanoparticles, it would be used to recover the copper present in the printed circuit boards. Therefore, the present study gives a way out to bioremediation as well as the recovery of valuable metals from E-waste in nano-form.

\section{EXPERIMENTAL METHODS}

\subsection{Preparation of plant extract}

For the preparation of plant extract of Cassia auriculata, $25 \mathrm{~g}$ of leaves have been taken and washed thoroughly in distilled water. The washed leaves were dried after that it was cut into fine pieces. The crushed leaves were added into $100 \mathrm{ml}$ sterile distilled water. Further it was heated at $60^{\circ} \mathrm{C}$ for 10 minutes and filtered through Whatman No.1 filter paper [9].

\subsection{Synthesis of Copper nanoparticles}

$25 \mathrm{ml}$ of leaf extract was added into $100 \mathrm{ml}$ of $2.5 \mathrm{mM}$ copper sulphate solution. The culture flask was then incubated at room temperature for $24 \mathrm{hrs}$. Reduction of cupric ions by leaf extract was observed by signatory color change (dark green color) which indicates the formation of copper nanoparticles. Further the reaction solution has been centrifuged for $15 \mathrm{~min}$ at $10,000 \mathrm{rpm}$ and dispersed in double distilled water to remove any unwanted debris [9]. 
2.3 Characterization of Copper Nanoparticles

The optical characteristics of the synthesized Copper Nanoparticles were recorded as well as confirmed by using Systronics 118 (UV-vis double beam spectrophotometer) by wavelength scan from $200-800 \mathrm{~nm}$ at a resolution of $2 \mathrm{~nm}$. To determine the functional groups present in the leaf extract, FTIR analysis was carried out which is responsible for the reduction of Copper ions with the spectral range of 400-4000 cm-1. FTIR spectrum was taken by NICOLET iS5 -Thermo Scientific, USA. The crystalline structure of the copper nanoparticles was determined by X-Ray diffraction spectroscopy (PW3040/60 X'pert PROPANalytical, Netherlands). The size and morphology of the synthesized SeNPs were analyzed using a Field Emission Scanning Electron Microscope (FESEM) (Quanta 250- FEG Co.Ltd) at an accelerating voltage of $10.0 \mathrm{kv}$. The spectrum of the energy dispersive absorption X-ray spectroscopy (EDAX) of the sample was carried out using an Oxford IE150 instrument.

\section{RESULTS AND DISCUSSION}

Cassia auriculata is an evergreen shrub that grows in many parts of India and in other parts of Asia. The flower, leaves, stem, root, and unripe fruit are used for treatment, especially in Ayurvedic medicine. We can use Cassia auriculata for diabetes, eye infections (conjunctivitis), joint and muscle

pain (rheumatism), constipation, jaundice, liver disease and urinary tract disorders. As a preliminary attempt we have been selected Cassia auriculata for the synthesis of copper nanoparticles.

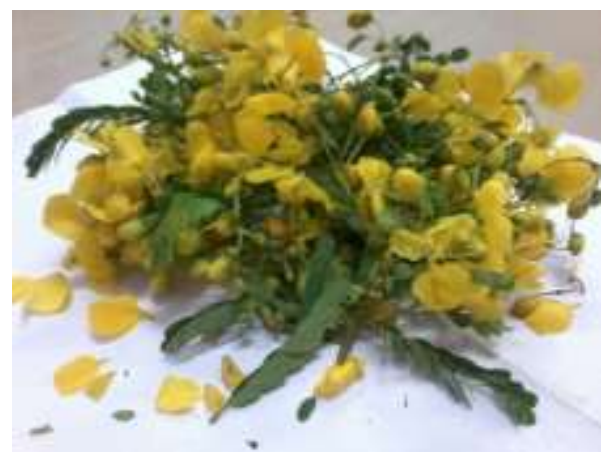

Fig. 1: Cassia auriculata

\subsection{Synthesis of Copper Nanoparticles by Cassia auriculata and its Characterization}

\subsubsection{UV-Vis spectroscopy analysis}

The optical characteristics of the synthesized nanoparticles obtained from UV-Visible spectroscopy analysis of the sample were presented in Fig 3. It is the most important method of analysis to detect the Surface Plasmon Resonance property of CuNPs [10]. The formation of CuNPs was confirmed from the peak at $300 \mathrm{~nm}$, this result of the present study similar with the results of Majumder, D.R (2012) [1] and the UV range was 300nm.

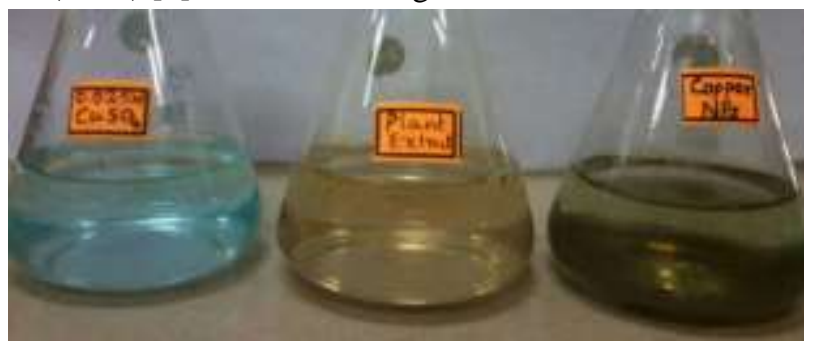

Fig. 2: Synthesis of CuNPs by Cassia auriculata

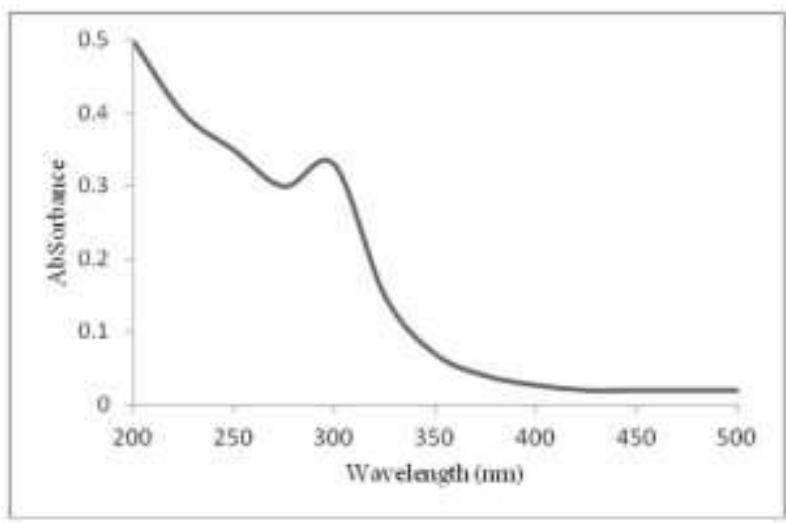

Fig.3: UV-visible spectrum of CuNPs

\subsubsection{Fourier Transform Infra Red Analysis}

The FT-IR measurements were carried out to identify the presence of biomolecules responsible for capping and efficient stabilization of copper nanoparticles synthesized by leaf extracts of Cassia auriculata. The infrared peaks were ranging from $1000-4000 \mathrm{~cm}^{-1}$ and thus confirmed the presence of polyphenols with aromatic ring [9].

\subsubsection{Field Emission Scanning Electron Microscopy and Energy Dispersive Spectroscopy Analysis}

The size and surface morphology of the copper nanoparticles were obtained by Scanning Electron Microscopy (SEM) analysis. The Figure 4 shows the CuNPs synthesized by the plant extract of Cassia auriculata. 


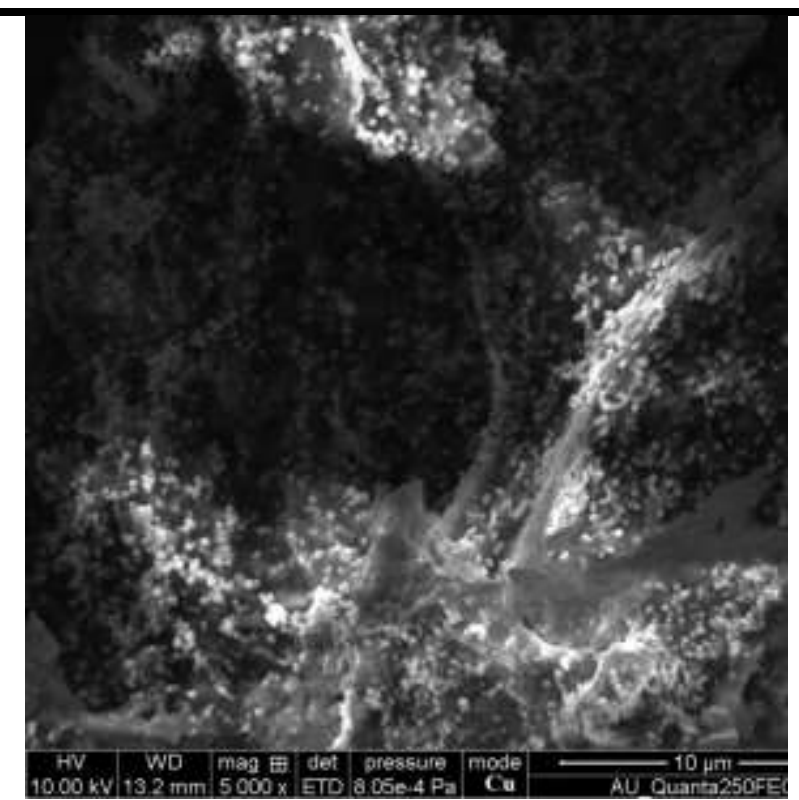

Fig.4: FESEM image of CuNPs

The electrostatic interactions and hydrogen bond between the bio-organic capping molecules bond were responsible for the synthesis of copper nanoparticles using plant extract [9]. It was shown that spherical and relatively uniform shape of the copper nanoparticles was confirmed in the range of $50-100 \mathrm{~nm}$. The results obtained from EDAX strongly confirmed that the synthesized nanoparticles were copper nanoparticles without any peaks of impurities [1].

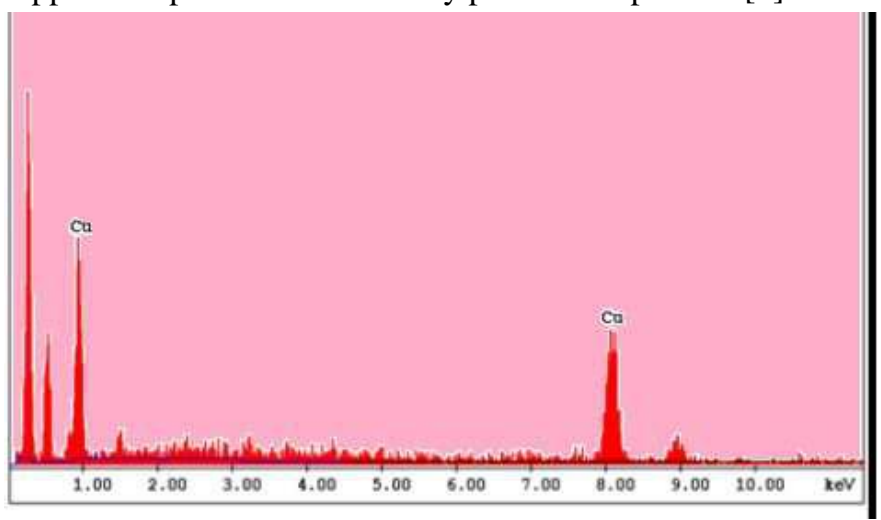

Fig.5: EDAX analysis of synthesized CuNPs

\subsubsection{X-Ray Diffraction Analysis}

The crystalline phases of the synthesized copper nanoparticles were confirmed by the characteristic peaks observed in the X-Ray Diffraction image (Figure 4). The diffraction peaks were observed at $2 \Theta$ angle and it was indexed as (111), (200), (220), (311) and (222). Similarly in the studies of Majumder D.R (2011)[1] and Abboud et al (2013)[12] the peaks of the copper nanoparticles were indexed as (111), (200), (220), (311) and (222). Hence the result of the present study strongly revealed that the synthesized nanoparticles were Copper Nanoparticles.

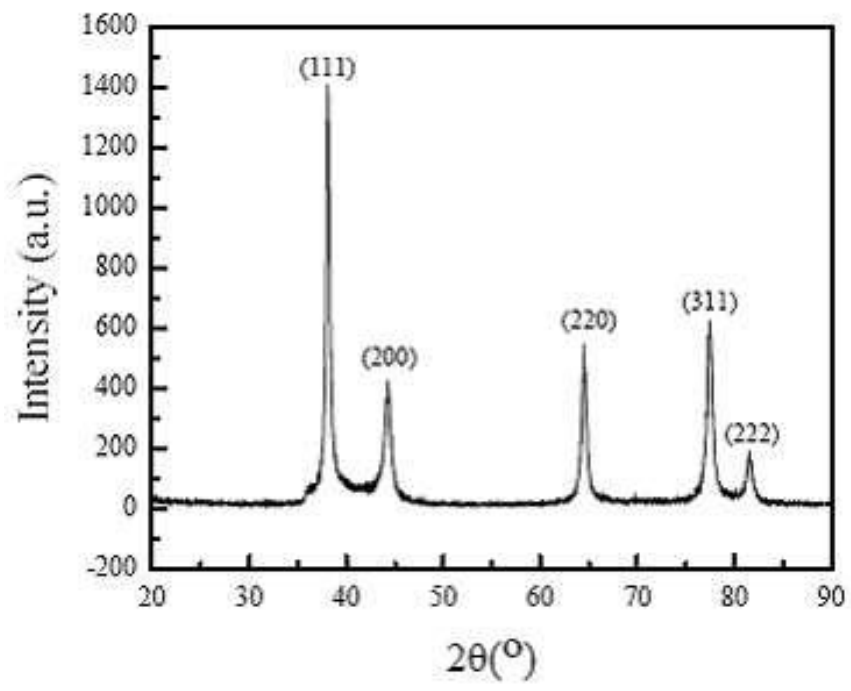

Fig.6: XRD pattern of synthesized Copper Nanoparticles

\section{CONCLUSION}

The present study is a preliminary investigation to know about the capability of Cassia auriculata to synthesize copper nanoparticles from its salts. The results obtained from the characterization techniques confirmed that the leaf extract of Cassia auriculata be capable of recovering copper from printed circuit boards in the form of nanoparticles in near future. Hence the present study is going to be a solution to bioremediation as well as the recovery of Copper nanoparticles from Printed Circuit boards.

\section{REFERENCES}

[1] D.R.Majumder. Bioremediation: Copper Nanoparticles from Electronic waste: International Journal of Engineering Science and Technology, 2012, pp- 43804389.

[2] B.Eswarapriya and K. Jegatheesan,.Antifungal Activity of Selenium Nanoparticles Synthesized from Electronic waste. Int. J. PharmTech. Res. 2015.

[3] Cui, J., Zhang, L., Metallurgical recovery of metals from electronic waste: A review. J. Hazard. Mater. 2008, 158, 228-256.

[4] Kiddee, P., Naidu, R., Wong, M. H., Electronic waste management approaches: An overview. J.Wasman. 2013, 1-14.

[5] Narayanan, K. B., Sakthivel, N., Biological synthesis of metal nanoparticles by microbes. Advances in colloid and interface science. 2010, 156, 1- 13. 
[6] L.Rassaei, M.Sillanpaa, R.W.French, R.G.Compton, F.Markenv, Arsenite determination in the presence of phosphate at electro aggregated gold nanoparticles deposits, Electro analysis, 2008,20, pp. 1286-1292.

[7] D.Mishra and Y.H. Rhee, Current Research Trends of Microbiological Leaching for Metal Recovery from Industrial Wastes, Current Research, Technology and Educational topics in Applied Microbiology and Microbial Biotechnology, A.Mendez-Vilas (Ed). 2010, pp. 1289-1296.

[8] S.Gurunathan,K.Kalishwaralal,R.Vaidyanathan,

D.Venkatraman, S.R.Pandian, J.Muniyandi, Biosynthesis, purification and characterization of silver nanoparticles using Escherichia coli, Colloids Surf B: Biointerfaces, 2009, 74(1), pp.328-335.

[9] Saranyaadevi K., Subha V., Ravindran R.S.E and Renganathan S. Synthesis and Characterization of copper nanoparticles using Capparis zeylanica leaf Extract. Int.J.Chem.Tech, 2014, 6(10), 4533- 4541.

[10]P.K. Khanna P.K, Gaikwad. S, Adhyapak P.V, Singh $\mathrm{N}$, Marimuthu R. Synthesis and characterization of copper nanoparticles: Materials Letters 61 2007; 4711-4714.

[11] Abboud Y., Saffaj, T; Chagraoui, A; Bouari, A; Brouzi, K; Tanane, O; Ihssane, B. Biosynthesis, Characterization and antimicrobial activity of copper oxide nanoparticles (CONPs) produced using brown alga extract (Bifurcaria bifurcata). Appl Nanosci 2013; DOI 10.1007/s13204-013-0233-x. 